\title{
COMUNIDAD ACADÉMICA: UN ESPACIO DE INTERACCIÓN DE SABERES
}

\author{
Por: Comunidad Académica Cuerpo \\ Movimiento \\ Jullefte Agámez Triana \\ Bellazmin Arencs Quintana \\ Hernán Restrepo Barrero \\ Jorge Elléeer Rodriguez Giraldo \\ José Hoover Vanegas Garcia \\ José Armando Vidarte Claros
}

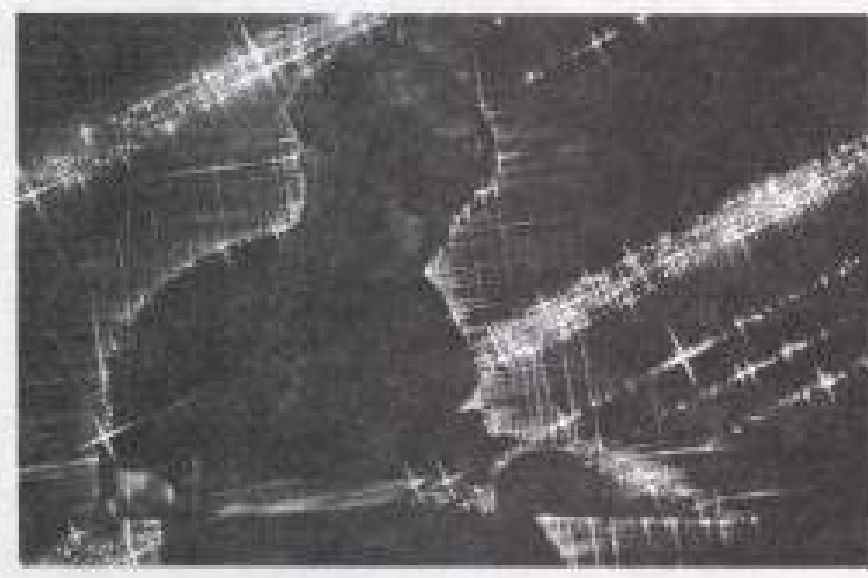

\section{NTRODUCCIÓN}

La bisqueda dol onnocimionto siempre ha sido un elemento ceniral en todas las culturas, y especificamente en los individuos, buscar el conocimiento significa desoeultar las causats o los motivon de todns los procesos que encontramos a nuestro redodor y aùn de nosotris mismos, tanto como corporalidades cósicas que como cuerpes animiens y aum como seres sociales. E conocimiento es fa condieión en la cual iodos los seres humanos, de uma o de otra fonna estamos inmersos,

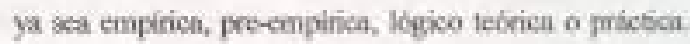

Ahora bien el conocimiento es un aeción en donde sedan dere dicmerstes: el hombre que se dirige " $a$ " y al " $a$ ", lo conocido, lo primero es al sujeto cognoscente y lo segundo es ci objeto conocido, a esta acción se lo llama vivencia, es un darse del hoenhre al mondo para significar y resignifiear of mismo, en este sentido, el producto del ecenceimicnta es la creacion de nuevos significados, a saber aucvas forma de ver, entender e interpretar el muisdo. De acuierdo con eato, ef conocimiento hace parte del ser del hombre puesto que ser es hacer y en of haoet sx 
formin el ser. El conocimiento es en el homber poe naturalerx, es su propia esencia

No obstante, el conocimiento no sulo es un proceso individual, más en las áltínag décadas en donde este ba aumeatado en forma desorbitunte, Esto quiete deeir, que en la actualsdad es mposible que un solo individuo mbance il opnocer todo lo que cxiste, por esth es necesario ta consolidación de grupos que se encuesten en un fin determinado pero desde varios ingulos del saber, es decir, la convergenein de varias vivencias en la básqueda de un solo objetivo, a esto le podernos llamar inieialmente Comunidad Acedénica, De acuerdo con esto, este trabajo pretende moatrar o lematizar el concepto de Comunidad Achdernica de acuetdo a sus caracteristicas màs importastes.

\section{COMUNIDAD ACADÉMICA}

Una Comunidad Academica as puede entender, imicialmerite, eceno un ecajunto de indivydues que somparten un mismo fin, pero además exmparters un fragrealo de exisiencia, es desir comperten ticmpos y espacios, puesto que tienen los miancs intereses, en este sentido esta exposicson in vamos a desenibir en tres mornenlas, prumero el analisis del concepto de coenumsdad acndemica como tal; sogundo, la comminidad asademica como escenario de la acción comanicativa discarsiva; tereero, implicaciones mariles de la piesta en escena de la conumidad de saberes.

\section{A. EL CONCEPTO DE COMUNIDAD ACADEMICA}

En el programa de Inducción Docente de la Univetsidad Autonoma de Manizales refiriéndose al concepto de Comunidad Academica se dice; "Son grupos de persunas preferiblemente interdisciplinarios, solidamente formados, no necesariamente por el perfil acadénico de los miembros, por la convergencia de potencialidades, por el planteamiento de diversos prohicanas euryo proposito es. la apliesción, aprobación y validaciổn del conocimiento" Esta significa que una comuaidad esta integrada por un grupe de persoces lo cual quiere decir que es. un grupo de voluntados, de saberes prefenhlements diferentes, en este sentido, conanidad del latin communis (común). segnifica lo que no perteniece a nadie, lo que no es privative, esto es la que es de todos, lo que se comparte sin reclamar propiodad o pertenencia, comunidad significa en este sentide un grupo de personas que someten su voluntad a un fin comèn, come comènunión

Lo interdisciplinario implica la copartieipación de varias disciplanas vehiculizadas a un mismo punto, es la coexistencia de varios conocimientos particulares para interpretar un mismo tersa, pero desule varios oonocimientos, es decir, la comein-unión no sollo es de personat sano tambièn de conocimientos particulares. Pero también es la unión de potencialidades, es decir, de disposiciones frente al trabajo conjunto. Las propósitos como lo dice la cita anterior sorr primero, la aplixación de diebos conocimientos, a saber, In conexión entre lo feórice del conocimiento y la pràctiea del mismo, un eonhcimiento debe tener una forma de haoctla prixis, y onno tal debe tener un impacto on la vida, debe modifiear unos parametros o como minimo resignifiealos.

La apropiación es el segunds proposito que enuncia la cita en menciōn, aqui purece habe: una contravieción puesto que antes afirmábamos que la earacteriatica más importante de una comunidad acadénuca en no ser propiedad, si esto es verdad, entonex ¿qué signifiesmas cuanda anunciamos este propóaite? La Comunidad no pucdedepender de la egtadia de uno o de varios de las iniegrantes, en eide sentido elle ne puede ser propiedad, pero la apropiación como propósito es la pertenencia y el compromiso de cada uno de los individurs actives de ella. 1.a aptopiación consiste en seatisse eoma parie de la comunidad y de los unoocimicatos qoe en ella se desvelan

La validaciáa es el último propózito, este quiere sobresaltar, Ia seriedsed y la fundamentaceós de la parte del conocimiesto que estriten juego al intenor de una Cominidad Acadefrica, es decir, bacer lo que se debe para que los naevos conocimientos fengas garantia de ser serios e importantes para ia comunidad en genersi, en este sentido, les conocimientos que se trabajan en Ia comunidad debe exponerse cop pares académiens reconocidos en el fimbito nacosnal c miernacional, puesto que el onocimento gasna yalidez at interior de unta comunidad cientifica, esto se muestra, de acuerdo $n$ is cantidad de literatura que uparece en tomo al un problema erpecifien. $\mathrm{L}$. validacibn aparece vuander se reconoco pobliscancrite la serictad de un producto, y pam qoe esto se dé bene que haber un aval de pares neadémicos que ya tengan reconocimiento

Otros elementas que earacterizan una comunidnd académien son el intsrès, la inteneionalidad, y el objeta de estudin. Fl interés hace referencia a las preferencias de grupo, podemos afirmar que si bien hay 
isteresos particulares, estas no pueden oponerse a los intereses comunitirios, ef enterés de la comuinidad es la bateris que le da energin a toda la Comunided Acodémica, ahora bien, el interés debe ser pertinente, puesto que no todo concocimiento puede acr-abordado, ello depende de los fines de la comunidad misina y de sus propositam en el eampo del saber que une los intereses.

Ens euanto a fa iaterocienalidad, esta está dada en la possción que toma la oomunidud en la institución en doode se validan y se aplican los covocinientos; y recoande a la pregunta jeúles san las pretensiones de la coasunidad en cuanto a lo acadkmico para con la institucico? Lo eual debe eatar dado de aucerdo al benefeio cientifico y ln evolucion intelectual de la misma.

III objeta de estudio es punto nodal que neane los interesea y las intencionalidades de los participantes de la comunidad, este es it hrígula que determina el camino que se dehe andar, es gquien direceiona In investigación como tal, ademais el objeto de estudio er el punto en donde se encuentran lessaberes, este está determinado con antelación, poes la lahor de las ifisciplinas se detennina por el objeto de eatudio.

\section{B. COMUNIDAD ACADÉMICA COMO ESCENARIO DE LA ACCIÓN COMUNICATIVA}

La acción eomanieativa podemas entenderta como la actividad por medio de la caal kow serer humanos se entienden, se comprenden, se interpretan, es una actividud, paresto que la comanicación exópe en us interlocutor usa expresión ya sen finguistica o ontporal y nùn grática, esto es, no hay forma de comunioas sentimientos posiones o conocimientos. sino es a traves del cuerpo, en este sentido es unt acción. o aetividad, es comanieativa en el sentido de hacerle saber a otro it oteas suestras intenciones, opmunicar es com-partir, compartir un lugar comôn en una tesis o una afurnación, La comunicsción significa ta enpacidad que tenemos los seres humanos de instalamos en los comocinicutos, sentimientos y pasiones de las otro5, pero is In vez de ellon imtakerae on fos de nosotres:

E otro onocepto de la acción comunicativa es ti entendimicrito, este elemento implica variog eiementos phimero entenderse es liegar a un acuerdo, es decir, aceptar como valido el discurso del otro, o como minimo aceptar al otro como un interlocutor con derochos y deberes. en este sentido hay un meuendo tácito y es que el otro como yo tiene la capacidad de participar en el diseurso, en el fisilogo, de acuetdo con esto ponerse de acuerdo eon otro es aceptar las condicjones del atro y a la vez el otro aceptar nuestrascondiciones, per ello el entersimiente cs un acuerdo.

Otro clemento, no metos importante, de la acción comunicativa es th mzón, esta implica que los argumenlos que ne emites on un fiblogo deben ser tacionales, es decir, que teagan coliercneia lógica, que sear pertinentes y que protesdas validez foemal dentro de un eontexto acadérnico. Por último la noción comunicatrya exige la critica, a saher que easiquier afgunbento debe y puede ser expuesto al mablais, debe ger evaluado, y los interiocutores deben aeeptar tal valoracion estan expaesios a defender criticamente su argumento or if es el caso a replantesito.

Feniendo esto en cuenta podernos afirmar que la accion comunicativa ae de en el lenguaje, pero no en el lengoage corpenul, sino en é discurso lingilatico, ausque ecenplementado desde su intensión por la coxpresión corporal, ahora bien, todo discurno està elahomida por proposiciones y étas por actoe de habla, en ente sentida, el seto de habls es el eje de ls acción comumicativa.

Les actos de habla ticnea varios vectores que unifican su sentido, el eusl es comunicar, et primero de ellos es que todo acto de habla debe corresponde at un algo en el mundo externe de la naturaleza, no se tome oste concepto sólo deside lo fáetico o flisico, aina toda farma de existencia desde lo visible come los difholes, hasta les valotea, loe aentimientos, ko imaginado, lo recordado, es decir, todo to que de une un otru forma constituye un punto de reforencin para kos actos de habla. de acuerdo con esto un acts de hubla puede ser verdaders o falso de acuerdo a su reforente o de acuenko a lo que esta dirigido el acto de habla.

El lenguaje, coeno venimos advirtiéndolo, es otro clemento de los actos de habla, este como ya se dijo, es la berramionia de la cominicactón, y dethe tener cohereneia, es docir, debe ser conprensible. sato quiere signifiear la forma lógiea del diseurao para un interlocetor Este, el interlocutor que a la vez es un hablante, es el ofro elemento de los actos de habla, es decir, el enunciante y el decodificador, la categonis que debe posoer ef bablanto es la veracidad, a saber quo lo que enancia correspooda con lo que quiere decir, ea otroe petminos que no haya una intencionalidad escondida en lo que enumeia, sino que aca veraz en la información 
El vitimo vector que se une al acto de habih son las normas sociales, siempre deben existir unos criterios normativos ousudo se trata de juntar varias yoxusindes, esto es, cuando hay varias pogiciones frente aun tema, es recesario que exista un ente regulador, las normas son las que deternimum lo legitimo y lo ilegitimo de una sociedad en donde sus integrantes se encuentran en el arto de habla, es decir en loe argubentas, o si we quicte en el discurva.

Este es el escenario en donde se personifica la comunidad acudémica, cada une de estos elementus detoc desempeîar un papel fundamental al interiot de dicha comunidsud académica Las intereses colectivos deben tener supremacia por encima de los iatividuales, is intencionalidad debe suplif las necesidadex intelectuales, no solo de b institución en dornde ella actúa sino, a nivel bacional $\mathrm{e}$ internacional eomo un impacto académico que contribuye al bienestar de la humanidat

La racionalidad y Is eritica deben set. igualmenie, elementos que siempre estén firesentes en el escenurio de la comarsidad scadémica, lo mismo que las vectores del acto de habla: la verdad, la comprensibitidod, la veracidad y la legitimidad, son elementos que ie dan solidez y estabibiad al prupo de persoeas para consolider nuevas conocimientos. Str embargo, hoce falta selarat gque no todo diseurso es argumentativo, teneinos que penarar en la laber que desempeîa el discurso retótico al interior de tona comanidad.

El discuran retoícico no pretende demestrar. gino seducir, es desir que los angumentos de este discurno no son contrastables con la realidad, sano en fa emocionalidad, en estecaso los argumentes an juicios de valor, of se quicte subjetivos, no abstante, es indispensable dentro de la Comanidad Acadermica. paesto que las interneciopes humuans iso estatn hechas soblo de los enanctados objetivos, quo es un dascurso frio, sarente de calor bumano, el amor por ejemplo, está elsborado con cnurciado de este tipo y soria impositle hacer enunciados objetuvos sobre este-lema, ef discurse reborico ie permite a li comunidad eaconfrarse en aul sentimientos, en mas pasiones, en ste ser humanos.

E acto de habla no sollo habita en el discurso argsumeatativo sino tambicti en el discurso retsinio y ambos conforman ta aceib́n tacional eomunicativa, fundamental en la interacción de los participantes de la comunidad académica. Ahorn bien la acciôn racinnal comunicativa posec tres categotias: la teleológiea, la normativa $y$ la dramatürgica La categoria teleologica implica que todo diseurso, llamese argumentativo logieb o retorion, siempre tiene un fin, un telos, esto es, un pusto de Hegada, en este sentido toda aceión de la comunidad debe llevar una intencionalidad, la que exige el fin acadénico del grupo

En cunsto a ia eategoris de normatividat, podemos agregar que todo dscuno norma, es decir, tode acto de habia fibera u unas acciones y prohibe otras, en este seribido ol discurso siempte buser eambiar una actatud en les intezkeuloecs. Aai al interior de una eomunidad is diacusion intetectual es en ella misma normativa, hay temas que requieren de una rigidez más profiunda que ctras y como tal se debe nomativioar la labor de eada une de los integrantes de la misana, lo miamo se puede afirmar de los campos del saber y las respoesabelidades de quaen los maneja y sa aporie al prupo.
Por Gltime, is labor de fa drumaturgit al interojor de la ueción meional eormunicativa es la de contribuir a la comprensión del diecurso medianle la personifieación eorporal de lo que se diee eon el euerpo, es decir, in dranaturgia es lo visibie en el cuerpo como carmpo expresivo del diseuras, de acuerdo oon esto, la comumidud académica debe cjercitur todos los elementos Sin embargo, esto no es suficiente pues hace falta para in cansolidación de una conmunidad alganss aspectes morales como intenthremes mositrirlo a contimasción.

\section{LA COMUNIDAD ACADÉMICA Y LOS VALORES MORALES}

Los valates se manifiestan corno intereset sobresalientes al interior de uma cultura, esto Es, son valencia o apeciaciones a fos ciales toda persona meional debo inclinarie, ellos le permiten a una comunidad su estabilidad monal, tomamos ef termino moral en seatido del hacer disrio de los seres humanos en donde se pone en juego la dignidad de los otros, es decit, fodh tocion que involuerd el ser personia de lo demas, por esto es indispensable pensar en qué valores momales deben sobresalir en una cornunidad coma un grupo de voluntades con preferencias y conceptos diferentes De seuerdo a esto vames a instalamos en los siguidentes valores la fibertail, la iguaidad, la sotidaridad, el respeto y el dialogo y ellos vistos a in ler de is justicin.

La libertad, es la facultad para tomar dectsiones racionales de acuerdo a un fin, todo individuo taene ia facultad de tomar decisiones, esto es lo que hace $\mathrm{n}$ los serea humanos racionales, poder elegir entre 
cumplir una regla o violarla. I a autodeterminacsón de la voluniad es a to que se le ha lismado labertad, pero sata depende de las reglas. En eate sentido la fibertad se expresa en el mundo de tres formas: la autonomia, la independencia y la participacoón.

Las reglas en fanto limites que exhortan un acto y prohiben otros, son determinadas eime correctas in eontrityyen al bienestar humano y a la felicidad individual sin oponerse a los fines do les demás, o inesrrectas si obstacalizant el bienestar humane. En este sentido podemos entender el valor de la autonomia, como el interes de un individeo por las reglas correotas de acuerdo a su propio fin. La sutanomia es is eapacidad de la voluntad de vivir de scuerdo a sus propies reglas sin violar las normas de una colectividad

La istoboutia como un valor que se deriva de la libertad posoe tres caracteristicas " fursdamentales, como minimo, primero la autenticidad, esta cualidad de la autonomia implies una cierta identifiesciòn con la eleceiber de las reglas que van a regir el comportamsicnto del individıo, pero tamhien la elabonaciòn de nuevar reglas en tuato no viole los derechos y los deberes de los otros. La autentictdad es una cualifad de la autonomia puesto que debe cumplir estos purimetros y lin embargn, lanto en vu elección cosno en su formación tiene clementos creatives, No obstanse, ests auteribicidad se ve debejitada caando una alteravida de las reglas moniles interviene en mu cjecución.

Segundo, La reflexión nucional, esta cancteristica denota en los seres monales tads la faceltad pre-dada, el analisis de las impresiones de acuerdo con el fín de la secion misma. La neicenalidad implies poner en euestion las consecuencias de la acción de acuerdo con la conciencis del agente ejevilante de fos acins en tanto la integridad de la otredad La astonemis en este sentido implica is inclinacion per las reglas y principios ecrrectos en faver del onden justo de una conunidad, eomo

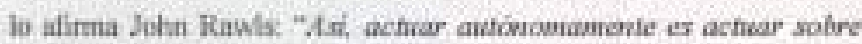

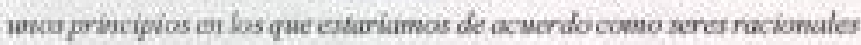

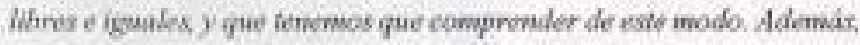
estas prinapios sou objumas. Son bos pirincipias que deseariamos que hodes finchidos mosotros misnasl siguícramos, aungue sólo fuena pana adopher en convint al panto de visfa goveral adecuado"
Ahora bien, la autonomia llevs en si mivana la elecoion racional de lo justo, peto ello impliea que existan otrus elecciones: lo injesto, to imparcial, o al contrano la elección de la parcialidad implica que exista algo injusto.

Tercero: la fuetza de voluntad, Para que esta caracieristica de la uutonomia se cumpls es necenaria la cualidad antes expuesta en el ejercicio de la fibertad. Aunque la volunitad; como la fuerna del deses por ejecutar unos actos y no gjosutar ofron, lleva implicito en carbefer peicológiog, también podemos verlo exno la cronsolidación del carieter por metio de los hábítos buenos que en últimas se convierten en costumberes, por eso la personalidad es lo que se ha conquistado en las vivencias misanas, como lo afirma Arangurea. ".. El enhdefer constituye ina inpresión de nasgor en la persovin misma: el candicter es fa personalidad que homor conquisfado a traves de la vida. lo yee hemes thecito de nasouros mismos viviendo"t?

La fuerza de voluntad depende, en muchos cased, de la habitualidad de la personalidad de los individuns y atasque ella constituye la resistencia del indrvituo por lo tacil, lo agradable, en lanto incornecto. debemos entender que no todo lo facil y lo agradable es incorrecto, en este casa es to que esté en contra de la humanidad. La fuerza de voluntad consiste en la eapacidad de los seres humaros de inclimarse por las reglas que coatribuyan al bienestar de la humanidad.

La independencia cobra valar en la medida en que it sujeto sea an indrviduo autonomo, ber independiente no sigsifiea tomar deceisiones a la deriva, sine elepir sin nimguna restricesón exferior a et, peto sc diferencia de la utotonotnis porque ésta consiste en la facultad def sujeto de ssberse únicat e irrepetible, Aentro de unu cormunidad de uaiformidades. La participacion hace referencis a la posabihdad bbre de cualquier sujeto de formar parte do la toma de devisiones er lana cotrunidad, el valor de la participación es una apreciación domocratica fundaninatal en la torna de decisiones

Del concegto de libertad podemos derivar el valor de iqualdad, ente valor de gan importancia imploes que fodse los seres humanos tiene los mismos derechos y los mismos deberes, es decir, las mismas

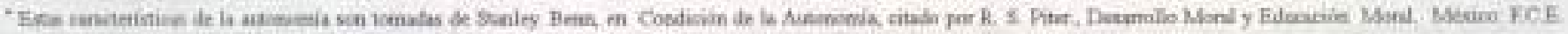

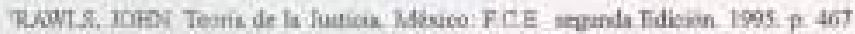

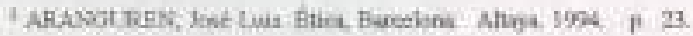


oportunidades en un sistema politico demueritien.

La selidaridad esta emenarcada dentro de los fines, ella no significa ayudar en un ahorn, sime en contribiair a los fines de la combenided ea general, la setidaridad es la contribacióce de los individuos a un solo fin que contribayn al bienestat de la comanidad acedénica, en este casw, es decir, toda catnunidad debe tener uns fines consobdados democráticamente, pers tambien hay fines individuales, cada persons busela la felicidad y ćsta se da en la medida en que los fines individuales se eumplen. La wolidaridad consiste en un acuerdo eatre Ins fines individuales cob los fines institucionales, $y$ a fa inversa.

\section{El respete va unido a Ia responsabilidad y a}

Is hanestidad, en realidad cuanda se respeta se es reaponsable $y$ ambos incluyen ta honestidad; la comprensión del otro con sas fro-juicios; omaptender, ne segnifice estar de actuerdo wen sus posicinoes, en la medida en que seun posiciones roluntarias, comprender signifiea interpretar lax intencionalidades ajenas, no cerrarse destro de su propia pereepeión, sine abrit la puerta de is intercomunicación, y acepter las diferencias, sill accptar en muches casos las decisiones de los otros, pero es que se puede estar de acuerdo con el ctro, sin aceptar las podiciones del miamo, esto es, se pueden crear acuendos entre los desacuerdios en la medida en que no agroda la iniegridad de níaguno do kas actores.

La bibertsd, fa igualdad, la solidaridad $y$ el respeto son valorea que te develan y se legitiman solo en la comunicación, en otras palabras, Jos valores soblo se ejecutan en las roliciones de las seres humanos y éstas sólo se dan en la conumicacioin. Fl dialogo es una forma de comuricación avanzado que han desarnollado los serea racionales, el diálogo como valor signifies, etirnologicansente: di; dos o comomínime dos, y lages, razón, argumenta. El salogo es una de les formas máximas de la comumicacionn, es decir de las interaccioties hamanas, en donde el objetivo er el ecovenio.

Dentro del distogo toda persuna tiene ef derecho y el deber de participar dentro del discurso, puesto que se considera que la verdad no essabjetiva, ella ex como minimo a dùo, de aqui podentos inferit que eeslquier persona tiene dereobo de participar con razones y como un igual dentro de las decisiones que se tomen, eate valor tiene comuoteciones polities puesto que el criterio democrático exige la daseusión argumentada y la conclusión debe ser el argumento que beneficie a la mayor cantidad de gente pasible.

Precisamente el valor de la justicia consiste en esto, en reoonscerte a cuida ser humano su aer persona: libfe, autónomo, igual, solidario, respetunso $y$ dispiaesto a participar on las decisionen que sean necesariau La justicia es la valencis que debe scompaniar todos los valares, la justicía entendida en sus tres dimensiones: distributiva, enamutativa y legal. La justicia distributiva inplica darle a todo el mundo pot igual el recenocimiento de ser, esta forma de justicia es la que nombrátamos en el pairmfo anterior, la jesticia conmutativa hace referenciâ a la distribución de tributos y beneficios los cualea no son por igual, sine de acuerde al mérito que un sujeto o tana comunidad tenga; $y$ la legalidad de la justicia signifien la obodiencia de la ky de scuerdo a sus disposiciones constitucionales.

De acuerdo eon esto una cominidad académica debe tener en cuenta lanto la bibertad como la igaaldad, la sobidarided, el respeto y el dialugo ya g̨ue como Comunidad debe sobrevivir a los obstáculos a los que todo grupo do persunas debe ezifrentarse, y en este caso con mayor importancia poesto que el fin es Acadénico, esto puede significa! que por encima de los intereses particulares estin los fines colectivos, dobe haber unu suprerracis en donde seoesidere la existencia de kos otros como perootses econ dignidad y no come instrumentes dof conociniento.

\section{BIBLIOGRAFIA}

ANGUREN, Jose Luis. Etica Burcelona: Alaya. 1994

CORTINA, Adela Ramón Counumicativa y Responsabilidad Solidaria Sulamanca: Sigueme, 1985. Ética sin Moral. Madrid Teenos, 1990

ECO Humberto y MARTTNL, Carlns Maria Eith que Cree los que no Croen. Un Dialoge Sober in Eticin en el fin del Milenio, Colombia. planeta. 1998.

FRONDIZ1, Risieri. Persamiento Axialógica, Colombia: Univernitad del Valle 1994

GUISAN, Fisperanza. Razoòn y Pasiỏn en Ética Scpunda Edicoxon. Barcelora. Antropoe 1990.

HABERMAS, Jargen. Escritos Sobre Moralidad y Eticidad. Bareelona: Paidos, 1993 
RAFHAEL, D D. Filosofls Moral, Mexico: Fondo de Cultura Econìmico, 1986.

RAWLS, John. Teoris de la Justicia. Méxios: segunds edición: FC.E 1995.

SANCHEZ Vazquez, Adolfo, Etiea. México: Grijalbo, 1969.

SAVATER, Fermando. Éticn oomo Amor Propio. Barcelona: Grijalbo 1995.

VANEGAS, Jook Hoover. HI Deber en la Etica kantiana Universidad de Calites, 1996
- Los Valores en in Vids Cotidiana; Universidad Autónoma de Manizales. Revista Aafora Nümero 14. Junis 1999.

La Étiea Un problema De Humaaidad. Inédito El estado y la soesedad civil a la laz de los nuevas simbolos normativos. Universidad Autonema de Manizales. Revista Anforn. Número 15. Jumio de 2000 